\title{
Guidelines to reduce sexually trasmitted infections at primary health care centres Part 2
}

\author{
STJ Molapo, M Tech Nursing student \\ Tshwane University of Technology
}

TMM Maja, D Litt et Phil

Senior Lecturer, Tshwane University of Technology

SCD Wright, D Tech Nursing

Senior Lecturer, Tshwane University of Technology

\section{Correspondence address:}

Dr SCD Wright

Tshwane University of Technology

Dept of Nursing Science

Private Bag x 680

Pretoria, 0001

Tel : (012) 382-5470

Fax : (012) 382-5033

E-mail : wrightscd@tut.ac.za

\section{Abstract: Curationis 30(4): 31-37}

Sexually transmitted diseases are the second highest cause of death among women aged 15-44 years. The incidence and prevalence of sexually transmitted diseases is on the increase at many health care centres despite their dreadfulness and many preventative measures. According to the Scope of Practice of registered professional nurses working in a community health clinic, the registered professional nurse has a responsibility to prevent disease and promote health as well as do research. This paper aims to describe guidelines to reduce sexually transmitted infections at primary health care centres based on research done to investigate the community specific knowledge, attitudes and perceptions regarding sexually transmitted infections. Structured interviews were used to collect data from 1694 participants (males and females) who were consulted at a primary health care clinic in Mamelodi, Gauteng province. Existing patients' records were also utilised to calculate the incidence rate of sexually transmitted infections. Data were computerised and analysed quantitatively to reflect the results in frequencies and percentages as reported in Part 1 of this research. Guidelines to direct the practice of registered professional nurse were developed based on the findings of the results. These guidelines must underpin community specific interventions to reduce the incidence of sexually.

\section{Background, rational and problem statement}

Sexually transmitted diseases are the second cause of death, excluding the human immunodeficiency virus, among women and men aged 15-44 years. Estimates are that 333 million new cases of treatable sexually transmitted infections, excluding AIDS and other viral infections, occur every year globally, and that approximately 11 million of these cases occur in South Africa (Mulick et al., 2001:37). The increasing incidence and prevalence of sexually transmitted infections pose a threat to public health owing to their long-term complications and the fact that they are co-factors in driving the HIV epidemic (Wilkinson et al., 1997:1).

This paper focuses on guidelines for the development of interventions that will reduce the incidence of sexually transmitted infections at primary health care centres. The guidelines are based on the results of the investigation into the incidence of sexual infection, the re- 
infection rate of male urethral discharge, and the knowledge, attitudes and perceptions of sexually transmitted infections at a primary health care clinic in Mamelodi, Gauteng province. Following the guiding role of the Health Promotinn Model suggested by Pender, Murdaugh and Parsons (2002: 68-80), the guidelines will be presented according to the propositions of the model.

\section{Problem statement}

The researcher has been working at the primary health care clinic where increasing numbers of clients seeking treatment for sexually transmitted infections have been observed, hence the urge to conduct this research. Even when excluding HIV/AIDS, the clinic's statistics indicated that the incidence of sexually transmitted infections increase from less than 2000 to 3782 for the period from June 2003 to May 2004 when compared to the previous period. Though health education is a standard aspect of the clinic's interventions to reduce the incidence of sexually transmitted infections, the methods used, the message or the approach were not successful in increasing the knowledge of the community members. Increasing knowledge is the basis of behaviour change.

\section{Research question and objectives}

The following research question was formulated based on the research problem:

Based on the results of knowledge, attitudes and perceptions of clients and national and international literature, which guidelines could be developed to enable registered professional nurses to reduce the incidence rate of sexually transmitted infections in clients at a primary health care clinic in Gauteng?

The objectives of the study were to develop guidelines to reduce sexually transmitted infections in a clinic in Gauteng to assist registered professional nurses in reducing sexually transmitted infections.

\section{Research design and method}

A contextual quantitative survey design (Burns \& Grove, 2005:36) was used to measure the knowledge, attitudes and perceptions of the clients using the primary health care clinic. The target population was clients (15 years and older) using the clinic as a health service resource during a pre-determined three month period. The sample size realised as $1694(n=1964)$. The data gathering method was self-report using structured interviews. The questionnaire was constructed based on previous instruments (Diale \& Roos, 2000:138; Reddy et al., 2004:265; Khoza, 2004:39) and the theoretical basis of the study. Based on the Health Promotion Model, several propositions was included, namely prior related behaviour, perceived benefits of action, perceived barriers to action, interpersonal influences, situational influences, immediate competing demands and a commitment to a plan of action. Data from the completed interviews were computerized and calculations made in terms of frequencies and percentages.

\section{Validity and reliability}

Experts in sexually transmitted infections and quantitative research assessed the face and content validity of the instrument. Comments made were addressed. The instrument was also pretested with 20 participants who met the sampling criteria and these participants did not take part in the main study. The fieldworkers were registered professional nurses and trained to gather the data consistently over the three month period.

\section{Results}

Owing to the pervasive influence of sexual health and sexual relationships in a person's life, specific propositions of the Health Promotion Model (Pender, Murdaugh \& Parsons, 2002:68) were used as the basis for the guidelines. The Health Promotion Model proposes 15 propositions which are theoretical statements with regard to health behaviours. Specific propositions will be used as the basis for the guidelines to reduce the incidence of sexually transmitted infections at the primary health care clinic. The applicable propositions include:

- $\quad$ Prior behaviour, inherited and acquired characteristics and personal beliefs influence and affect the enactment of healthpromoting behaviour.

- $\quad$ Persons commit to engaging in behaviours from which they anticipate deriving personally valued benefits

- $\quad$ Persons are more likely to commit to and engage in health- promoting behaviour when significant others model the behaviour, expect the behaviour to occur, and provide assistance and support to enable the behaviour.

- Situational influences in the external environment can increase or decrease commitment to or participation in health-promoting behaviour.

- Commitment to a plan of action is less likely to result in the desired behaviour when other actions are more attractive and thus preferred over the target behaviour.

- $\quad$ Families, peers and health care providers are important sources of interpersonal influence that can increase or decrease commitment to and engagement in health-promoting behaviour.

Guidelines will be presented in view of the proposition, the findings of the study and the proposed guideline to improve the current situation. Relevant literature control was done to verify the results.

\section{Proposition 1:}

Prior behaviour, inherited and acquired characteristics and personal beliefs influence and affect the enactment of health-promoting behaviour.

Results of the study related to the proposition:

- $\quad$ Slightly more than half $(53.3 \%)$ males visiting the primary health care clinic on a regular basis were below 40 years, and $10 \%$ were under 21 years of age.

- $\quad$ Though the incidence rate of sexually transmitted infections was $6.3 \%$, the age-specific incidence rate varies and any intervention to reduce the incidence rate should focus on those aged 15-20 and 21-40 years.

- The re-infection rate of male urethral discharge in the study sample was $10.0 \%$.

- The 15-20 age group are at particular risk and would need age-specific strategies to reduce their risk of acquiring a sexually transmitted infection.

Concerning homosexuality, more females $(85.7 \%)$ than males 
were reportedly homosexuals.

Guidelines based on proposition 1:

All age groups should be targeted for reduction of sexually transmitted infection interventions. The interventions should be ageand gender-specific. The targeting strategy should be culturally sensitive, especially among the young people who have their own culture which they utilise to learn new matters. Targeting is the process of designing and building an intervention programme on the basis of the intended audience segment (Singal \& Rogers, 2003:169).

- The appropriate learning and teaching approach should be used for the various age groups. Due to the unconcerned attitude of the younger group, teaching methods based on adult education would not be suitable for this group. If they are unconcerned, they will not have a need to know and thus they will not be teachable at that moment.

- $\quad$ In targeting the young people, their programme should include life skills that include assertive behaviour, problem solving and negotiation skills to encourage their confidence and self esteem.

- $\quad$ The Love Life youth centre should be strengthened to meet the needs of young people with regard to sexual health.

- $\quad$ Mehta et al. (2004:128) recommended that, due to the large proportion of patients who experience re-infection, patients who have a high risk for infection should undergo routine screening.

- Specific factors associated with an elevated risk for re-infection suggest possibilities for focused prevention interventions at the first diagnosis of sexually transmitted infection. Targeting interventions for people at risk for re-infection may be an effective and optimal way of preventing gonorrhoea transmission and reducing resource utilisation.

Possible strategies to prevent sexually transmitted infections should be discussed with the patients. The strategies include masturbation, exercise, circumcision, infibulations, shaving, vaccination and thigh sex. These strategies could be exploited to complement existing public health programmes and can be helpful as they are known to the community as they have been practised for many years. Individuals often make decisions by themselves or under the guidance of health professionals, family, peers or significant others. Perceived self-efficacy in the prevention of sexually transmitted infections with these methods could promote their use, especially in young people. Increasing the youth's perceptions regarding selfefficacy should be a priority and may empower them in preventing sexually transmitted infections.

- The number of re-infections in the 15-20 and 21-30 age groups indicates a norm with regard to the number of sexual partners and risky behaviours. Agespecific interventions to change these norms should be researched and implemented. Clarke (2001:251) suggests that nursing must evolve from sick nursing to health nursing.

Health nursing focuses primarily on the promotion of health in the community by creating health partnerships and support changes to healthy lifestyles and well-being.

\section{Proposition 2:}

Persons commit to engaging in behaviours from which they anticipate deriving personally valued benefits Results of the study related to the proposition:

- The majority of the participants (92.6\%) claimed to be informed regarding sexually transmitted infections. However, when asked about the transmission, signs and symptoms, types and complications of sexually transmitted infections, lack of knowledge was evident.

- $\quad$ Almost a third of the group (36.6\% males and $25.9 \%$ females) were not influenced by the risk of HIV/AIDS when contemplating a sexual encounter.

Though the group knew about prevention methods, preventing sexually transmitted infections was not part of their decision making when considering a sexual encounter.

- $\quad$ The value attached to a sexual relationship is presently a barrier or competing demand to health-promoting behaviour.

- The majority of the participants $(79.1 \%)$ indicated infidelity to their sexual partners.

- Most participants were informed about the location and availability of condoms.

- $\quad$ Condom use was reported to be low and inconsistent and negative attitudes prevailed regarding condom use.

Negative attitudes may be barriers in the challenge to reduce the incidence of sexually transmitted infections in the community surrounding The primary health care clinic.

\section{Guidelines based on proposition 2:}

- Participants' evaluation of their own level of knowledge regarding sexually transmitted infections might not reflect their real level of knowledge. A single question regarding level of knowledge will not suffice to inform the health care workers on the knowledge of the patient. Lack of knowledge is a barrier to engaging in healthpromoting action. Health education given in a knowledge vacuum will not empower the participant with regard to prevention of sexually transmitted infections.

- Knowledge is not as such, a valued goal for persons visiting the clinic or, by extension, for the community being served by the primary health care clinic. There is however, a desire to appear knowledgeable and this could be used as a starting point for educating the 
community.

- $\quad$ Though the personnel at the primary health care clinic have various programmes to educate their clients regarding sexually transmitted infections, no perception of vulnerability exists in the group. Personal vulnerability should be included in interventions to educate the community.

- The importance of using condoms consistently should be encouraged to clients. Health education should include demonstrations of condom use (Maja, 2007: 76). Information regarding the location of the availability of condoms should be made available throughout the community. Prior behaviour is a definite risk with regard to condom use and behaviour change should be encouraged through various methods. These messages should acknowledge the competing demands of immediate personal gratification, and replace risky behaviour with healthpromoting actions.

- $\quad$ Age-specific interventions should be developed for different age groups. Naude, Meyer and van Niekerk (2000:128) propose that patient teaching by means of demonstration is often an effective method of education.

Advocating of female condoms is essential, as in most clinics female condoms are a scarce commodity. Female condoms should be freely available in the same way as male condoms. The benefits of the use of the female condom could influence the participants to adapt to more health promoting actions. Commitment to one partner must become the norm throughout the community and multiple partners must be rejected to encourage young people to follow suite.

\section{Proposition 3:}

Persons are more likely to commit to and engage in health-promoting behaviour when significant others model the behaviour, expect the behaviour to occur, and provide assistance and support to enable the behaviour.

- $\quad$ The majority of (84.1\%) participants in the study had their sexual début under 21 years and that (18.4\%) of boys and girls have sexual intercourse for the first time at 15 years of age or even younger.

- $\quad$ Older males model multiple sexual partners, as well as failure to use condoms.

- In all age groups, some participants abstain from sexual relationships, with more men than women abstaining in all age groups.

\section{Guidelines based on proposition 3:}

- $\quad$ Young people should be taught the importance of postponing sexual activity until they are old enough to make meaningful decisions in this regard. Health education should be started in primary schools to prevent the early début of sexual interaction. Cowan (2002:318) recommends that interventions that combine a behavioural and structural approach will be those most likely to succeed, especially with the young people.

- The value of virginity and fidelity should be made desirable throughout the community. Accepting the status quo as 'culture' and therefore 'unalterable' negates all principles of health promotion; the services of the clinic will remain reactive in stead of proactive.

- $\quad$ Older males must be made aware of their function as role models and therefore specific interventions should be developed and tested to provide evidence for interventions of this nature.

More research should be done to gain an understanding of the group that abstains. This basis could possibly be used to popularise abstinence in the community. If abstinence is not modelled and accepted, young people growing up will not value it.

\section{Proposition 4:}

Situational influences in the external environment can increase or decrease commitment to or participation in healthpromoting behaviour

Results of the study related to the proposition:

- $\quad$ The majority of the group are heterosexual and the socioeconomic situation of just more than half of the respondents revealed that they are living in informal shelters, half the group were not employed and another $22 \%$ reported to be employed as unskilled labourers.

- Most of the study sample had a secondary or tertiary education and affiliated to different religions.

- The majority of the participants $(67.9 \%)$ indicated that they would support sexual partners on their being diagnosed with sexually transmitted infections.

- $\quad$ There were eight socio-cultural groups and therefore eight languages in the primary health care clinic, as well as foreigners.

- A low level of testing for sexually transmitted infections was reported.

\section{Guidelines based on proposition 4:}

- A multi-sectoral approach is essential to the curbing of sexually transmitted infections. Faith-based organisations, nongovernmental organisations, traditional healers and workplaces should be involved in the fight against sexually transmitted infections. The organisations and companies could be used to distribute free condoms. Traditional healers in Africa are often preferred to Western medical practitioners because they understand people's cultures and are more accessible to communities. Collaboration between Western medicine and African medicine should be encouraged to reduce the incidence of sexually transmitted infections. Simbayi et al. (2004:46) advocate that public health institutions, private doctors, traditional healers, schools and employees make the community more 
aware of sexually transmitted infections.

- When health education is conducted, any of the biases related to religious affiliations and types of employment should be disregarded as they do not necessarily indicate specific behaviours.

Stereotyping the client will create bias and the health care worker might consider clients to be knowledgeable when they are not. Biases result in lost opportunities for people to learn.

- $\quad$ Educational materials and programmes should be developed in the local language. Barriers preventing people from learning include language and level of education. Naude, Meyer and van Niekerk (2000:129) indicate language and level of education as possible barriers preventing people from learning.

- The level of literacy and socioeconomic situation of the group should be known when interventions are developed as the level of health literacy will influence the success of any intervention. Failure to use the appropriate language and culture-sensitive methods could also be a barrier to a successful intervention.

- $\quad$ Support for one's partner should include being tested oneself.

- The concept of being in control of one's health should be popularised. This type of intervention should include popularising knowing one's health status, knowing about medication and the difference between controlling a disease and curing a disease.

\section{Proposition 5:}

Commitment to a plan of action is less likely to result in the desired behaviour when other actions are more attractive and thus preferred over the target behaviour.

Results of the study related to the proposition:

The majority of the group were single and sexually active.

Homosexual relationships were more common among females than males.

- $\quad$ Multiple sexual partners are a reality in the community being served by The primary health care clinic.

- Less than one in five (18\%) of the participants did not routinely have a condom available.

\section{Guidelines based on proposition 5:}

- Behaviour modifications should be encouraged in the community; a wide spectrum of messages from various stakeholders should be coordinated. To overcome barriers to behaviour change, the healthpromoting interventions should focus on all aspects related to sexually transmitted infections, for example reducing the number of sexual partners and avoiding frequent partner changes and risk factors. The environment in the community should be conducive to people taking positive steps toward preventing sexually transmitted infections.

- The findings indicate that the majority of the participants were single. It is important that the three methods advocated by the government (abstinence, being faithful to one partner, and condom use) are promoted and well marketed in the community. The dual family planning method should also be promoted. The use of both condoms and other contraceptives will prevent both sexually transmitted infections and unwanted pregnancies (Maja, 2007: 71).

- Incorrect myths about promiscuity and frequent partner changes should be tackled directly and consistently.

\section{Proposition 6:}

Families, peers and health care providers are important sources of interpersonal influence that can increase or decrease commitment to and engagement in healthpromoting behaviour.

Results of the study related to the proposition:

- $\quad$ Asking if a person is knowledgeable about sexually transmitted infections and receiving a positive answer offers no guarantee that the person actually has any knowledge about the subject. More extensive questioning is needed to ascertain the person's knowledge.

- The concept of transmission might be poorly understood in the study sample and community surrounding the primary health care clinic.

The current methods of educating the community regarding sexually transmitted infections at The primary health care clinic are seemingly not effective in transferring knowledge and do not lead to a change in behaviour.

The low level of knowledge indicates a low level of health literacy that would also serve as a barrier to making healthpromoting choices.

- $\quad$ A group of the participants $(39.9 \%)$ indicated a sense of concern when thinking of sexually transmitted infections.

\section{Guidelines based on proposition 6:}

- Participants were concerned about readiness to learn and this indicated the ability to do something about sexually transmitted infections. Naude, Meyer and van Niekerk (2000:128) suggest that readiness to learn is a key factor in the success of any health education.

- Health care personnel at the primary health care clinic should investigate their practices of educating the community regarding sexually transmitted infections. Guidelines that could be incorporated in their practices are as follows: Health education on sexually transmitted infections should be taught by using movies, discussions, role-playing and visual aids. Simbayi et al. (2004:54) recommend using various media both in the community and clinics to improve the educational and counselling interventions. Using these media could 
enhance the aesthetics of the message in such a way that the uptake is improved.

Health education on sexually transmitted infections should be age- specific. When educating the older patients, pictures should be used to facilitate learning and different age groups should be taught separately from others owing to the sensitive nature of the topic.

Identifying and adapting health care interventions for homosexuals in terms of gender should be researched, and gender-specific interventions need to be developed and tested. Failure to be gender sensitive could be a barrier to teaching this group. The situational influence should enhance acceptance and caring; if not, teaching will not be successful.

Health promotion activities should have a culturally appropriate message at their centre. The activities may include discussion forums with local leaders and using informal opportunities at shebeens and sports fields. Traditional avoidance strategies of sexually transmitted infections could be used to curb the incidence of sexually transmitted infections (Donovan, 2000: 1; Mulaudzi \& Makhubela-Nkondo, 2006:55).

Health education should be done individually and in groups, especially after treating sexually transmitted infections. As part of individual counselling, the patients should be given time to air their problems and concerns regarding sexually transmitted infections.

Evaluation research should be done regularly to determine the success or failure of any health promoting intervention.

Health education is inadequate by itself but can become a key discipline that must be harnessed in any comprehensive management of primary health care concerns. Naude et al. (2000:127) state that health education should develop from a disease prevention approach to include the promotion of health.

Evaluation research should be part of the mission and vision of the clinic to ensure adequate learning and the highest standard of practice. An evaluation culture should be developed at all health care centres.

Reddy et al. (1999:1448) urge that there is a need for the development and implementation of health education policies and protocols including the facilitation of a social environment in which health education is actively supported and practised. Positive role models who champion and advocate health-promoting behaviour should be identified and used to transmit such messages.

African strategies to encourage people in preventing sexually transmitted infections have been developed (Airhenbuwa in Van Dyk (2004:127)). The strategy is termed the PEN strategy. Traditional beliefs and behaviour can be classified as (P) positive, (E) exotic and $(\mathrm{N})$ negative (Section 2.6.8).

Strategies known to be successful should be investigated for possible adoption in the local community.

- Leornard, Ndiale and Kapadia (2000:21) found that the use of peer educators in interventions to change men's attitudes and behaviours of longer duration seems to be effective. The peerbased model should be gender, sexual preference- and age-specific to be effective.

- Registered professional nurses should sit at a patient's eye level and use simple language to encourage comprehension during patient health education (Scudder, 2005). Written material should be continued but must be designed in visually appealing ways that will be accessible to clients with low literacy.

Ohnishi, Nakamura and Takano (2005:162) indicate that community literacy will improve

only if health education policy considers existing basic health care resources, living environment conditions and community activities. Staff should therefore include living environment, conditions and activities in the planning of health education programmes.

o

\section{Conclusion}

The importance of developing 
community-specific interventions based on the needs and problems of the community have been highlighted as a priority in managing sexually transmitted infections. The guidelines presented are developed in view of the results and the theoretical foundation of the study.

\section{References}

BURNS, N \& GROVE, SK 2001: The practice of nursing research: conduct, critique and utilization. $4^{\text {th }}$ ed. Philadelphia: WB Saunders.

COWAN, FM 2002: Adolescent reproductive health interventions. Sexual transmitted infections Journal. 78: 315318.

DIALE, DM \& ROOS, SD 2000: Perceptions of sexually transmitted diseases among teenagers. Curationis. 23(4), Dec.:136-141.

DE VOS, AS 2002: Research at grassroots. $2^{\text {nd }}$ ed. Pretoria: Van Schaik.

DONOVAN, B 2000: The repertoire of human efforts to avoid sexually transmitted diseases, past and present: Part 1: strategies used before or instead of sex [Online]. British Medical Journal. Available from: http:// sti.bmjjournals.com/cqi/content/full76/1/ 17 [Accessed: 29/06/2005].

LEORNARD, L; NDIAYE, I \& KAPADIA, A 2000: HIV prevention among male clients of female sex workers in Kaplack, Senegal: results of a peer education program. AIDS educational preview.

KHOZA, LB 2004: Adolescents' knowledge, beliefs and experiences regarding sexual practices. Health SA Gesondheid, 9(3), 34-41.

MAJA, TMM 2007: Involvement of males in promoting reproductive health. Curationis, 30(1): 71-77.

MEHTA, SD; ERBELDING, EJ; ZENILMAN, JM \& ROMPILO, AM 2004: Gonorrhoea re-infection in heterosexual STD clinic attendees: longitudinal analysis of risks for reinfection. Sexually Transmitted Infections, 79:124-128. healer's beliefs and practices concerning sexually transmitted diseases. Curationis. 29(1): 46-53.

MULICK,S; McCOY,D; BEKSINKA,M \& MOYS, A 2001: Evaluating the quality of care for sexually transmitted infections using district sexually transmitted clinic assessment. Durban: Gang.

NAUDE, M; MEYER, S \& VAN NIEKERK, $S$ 2000: The nursing manager: a comprehensive guide. Sandton: Heinemann.

OHNISHI, MO; NAKAMURA, K \& TAKANO, K 2005: Improvement of maternal health literacy among pregnant women who did not complete compulsory education: Policy implications for community services. Health Policy. 72(2):157-164.

PENDER, NJ; MAUDAUGH, CL \& PARSONS, MA 2002: Health promotion in nursing practice, $4^{\text {th }}$ ed. New Jersey: Prentice Hall.

\section{REDDY, P; MEYER-WEITZ,A; VAN} DEN BORNE, B; KOK, G \& WEITJTS, W 1999: The learning curve: health education in sexually transmitted infections in South Africa [Online]. Available from: file/l E:ISTI\%20healtheducaion.htm [Accessed:10/05/2005].

SIMBAYI, IC; STREBEL, A; ANDIPATIN, C; POTGIETER, C; RATELE, K; SHABALALA, N; SHEFER, T\& WILSON, T 2004: The evaluation of immediate behavioural outcomes of the syndromic case management approach for the treatment of patients with sexually transmitted infections at PHC centres in South Africa: knowledge, attitudes, beliefs and sexual behaviour. Journal of Social Aspects of HIV/AIDS Research Alliance. 1(1), May.

SCUDDER, L 2005: Words and wellbeing: How literacy affects clients health [Online]. Available from: file:// www.sciencedirec.com/science [Accessed: 25/04/2006].

SINGAL, A \& ROGERS, EM 2003: Combating AIDS: communication strategies in action, New Dehli: Sage.

VAN DYK, A 2004: HIV/AIDS care \& counselling, a multidisciplinary approach. Cape Town: Maskew Miller Longman.

WILKINSON,D; RAMJEF, G;STURM, AW \& KARIM, SA 1997: Reducing South African hidden epidemic of sexually transmitted infections. South Africa: Medical Research Council. 